ORIGINAL ARTICLE

\title{
Current practice in the management of anterior cruciate ligament injuries in the United Kingdom
}

\author{
B Kapoor, D J Clement, A Kirkley, N Maffulli
}

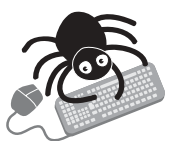

A copy of the questionnaire can be found at www.bjsportmed.com/ supplemental.

See end of article for authors' affiliations

.....................

Correspondence to: Professor Maffulli, Keele University School of Medicine, Trauma and Orthopaedics,

Thornburow Drive, Hartshill ST4 7QB, UK; osa14@keele.ac.uk

Accepted

17 November 2002
Br J Sports Med 2004;38:542-544. doi: 10.1136/bjsm.2002.002568

\begin{abstract}
Objective: To outline the current practice in the management of anterior cruciate ligament (ACL) injuries in the United Kingdom.

Methods: A postal questionnaire designed to include various clinical scenarios was sent out to the 321 orthopaedic surgeons in the United Kingdom who, being affiliated to one of the specialist societies of the British Orthopaedic Association, namely the British Association for Surgery of the Knee (BASK) or the British Orthopaedic Sports Trauma Association (BOSTA), have a manifested interest in treating such injuries.

Results: The response rate was $60 \%$ (192/321). Most surgeons diagnose and operate on less than $50 \mathrm{ACL}$ injuries a year. The following results were obtained: $58 \%(76 / 132)$ use bone-patellar tendon-bone autografts, whereas $33 \%(44 / 132)$ use semitendinosis/gracilis autografts; $84 \%(108 / 129)$ would not incorporate the $\mathrm{ACL}$ remnant in the reconstruction; $14 \%$ (19/135) would perform an $\mathrm{ACL}$ reconstruction in an 8 year child with an acute rupture; $30 \%(42 / 141)$ would perform an $\mathrm{ACL}$ reconstruction in a 14 year old with an acute $\mathrm{ACL}$ rupture.

Conclusions: There is wide variation in the management of acute and chronic $\mathrm{ACL}$ injuries among orthopaedic surgeons in the British Isles. Future research and randomised controlled trials should address the issues that this investigation has raised.
\end{abstract}

A nterior cruciate ligament (ACL) injuries are common sports injuries and one of the most commonly treated conditions of the knee in the young. ${ }^{1}$ Sports such as soccer and skiing account for most of these injuries in the United Kingdom. Although ACL injuries are common, there is still considerable variation in their management. If left untreated, this may lead to functional instability, secondary osteoarthritis, and increased risk of meniscal injuries. ${ }^{2}{ }^{3}$ There is controversy about the optimum methods of diagnosis, management, type of surgical procedure, and factors influencing surgical decision making. ${ }^{5}$ There are several graft choices for surgical reconstruction of the ACL. Bone-patellar tendon-bone autograft has been the most commonly used, but the use of semitendinosus and gracilis autograft is increasing. Although use of synthetic substances for ACL reconstruction has largely ceased, synthetic augmentation is still occasionally carried out. ${ }^{67}$

We felt that a national survey would help to establish the current practice of orthopaedic surgeons in the United Kingdom. This, in turn, may lead to the development of a unified approach towards the management of these injuries based on available evidence, and may also identify areas in which further research is needed. We surveyed 321 surgeons from the United Kingdom who, being affiliated to a specialist society of the British Orthopaedic Association, namely the British Association for Surgery of the Knee (BASK) or the British Orthopaedic Sports Trauma Association (BOSTA), have a manifested interest in treating such injuries.

\section{MATERIALS AND METHODS}

A questionnaire consisting of six sections (a copy of which can be found at www.bjsportmed.com/supplemental) was sent to the surveyed population. It was based on the questionnaire used by Mirza et al. ${ }^{8}$ The survey was designed according to recommendations on survey design by Dillman. ${ }^{9}$ Section 1 related to the diagnosis of acute haemarthrosis. Sections 2, 3, and 4 consisted of scenarios of ACL injury in acute, chronic, and failed conservative treatment setting. Section 5 consisted of various factors involved in decision making for ACL surgery. Section 6 consisted of data on the surgeon's workload, the higher surgical training scheme they had attended, and whether and where they had completed a fellowship.

The survey was mailed to the 321 orthopaedic surgeons in the United Kingdom who were members of the British Orthopaedic Association and belonged to one of two specialist societies, BASK and BOSTA. The addresses were obtained from the British Orthopaedic Association Handbook of 2000. The surgeons who did not respond to the first mailing were sent reminders at six weeks, and the remainder were sent faxed copies after a further six week interval. The results were scanned using Teleform (Cardiff Software) and were analysed using SPSS for windows (SPSS, Inc, Chicago, Illinois, USA)

The ACL injury scenarios presented in the survey were as follows.

- Scenario 1: A 22 year old university soccer player, with a five day old mid-substance tear of the ACL. There are no symptoms of a meniscal tear. He wishes to continue playing competitive soccer.

- Scenario 2: A 22 year old university soccer player with a ruptured ACL is seen one year after injury. There are no signs of a meniscal injury. The patient wants to continue playing competitive soccer.

Abbreviation: $\mathrm{ACL}$, anterior cruciate ligament 
- Scenario 3: A 22 year old university soccer player with a two year history of ACL insufficiency that has been treated conservatively has a giving way episode in a brace while playing a game. The patient wishes to continue playing competitive soccer.

\section{RESULTS}

The response rate was $60 \%$ (192/321). Twelve surgeons did not complete the questionnaire either because they no longer provided a knee service or they had retired. Most of the respondents (92\%; 166/180) would investigate a haemarthrosis and follow those patients on their own, and the remainder would refer them to another surgeon. Most of the latter gave the reason for referring the patient as being involved chiefly with knee arthroplasty work. Some 44\% (78/ 180 ) encountered less than 50 such injuries per year, and $73 \%$ (131/180) perform less than 50 ACL reconstructions per year. ${ }^{10}$ After determining the diagnosis of an acute ACL injury, $83 \%$ (150/180) would treat the patient themselves. Only 55\% (94/170) of the respondents had completed a fellowship. Some $43 \%$ (75/173) were attached to a teaching institution.

Acute haemarthrosis was predominantly diagnosed with the help of history, clinical examination, and plain radiographs. A few of the surgeons (2\%) use arthrocentesis. Magnetic resonance imaging (MRI) was routinely employed by $7 \%(12 / 180)$ of surgeons; $6 \%$ use it in private practice only, because of delays encountered in obtaining an MRI scan in the NHS. Most $(63 \% ; 114 / 180)$ would use MRI as a diagnostic tool if there was a possibility of associated damage to the menisci. Only $14 \%(25 / 180)$ of the surgeons routinely use arthroscopy for diagnosis of ACL injuries, whereas $71 \%$ (127) 180 ) would use it in the presence of an acutely locked knee.

\section{SCENARIOS}

In the first scenario of a 22 year old man with an acute ACL rupture, $58 \%(89 / 154)$ of surgeons would recommend ACL reconstruction, whereas $24 \%$ (36/154) would treat the patient with rehabilitation and bracing. Another 18\% (29/154) would advise rehabilitation for 5-12 weeks, followed by a reassessment. Of those who do recommend surgery, 30\% (39/128) would operate when the patient has full range of movement of the knee even if this meant immediate surgery; 50\% (65/ 128 ) would delay surgery even if the patient has full range of movement, and $8 \%(10 / 128)$ would operate irrespective of range of movement. A majority (57\%; 76/132) of the surgeons preferred to use bone-patellar tendon-bone autograft, whereas 33\% (44/132) would use semitendinosus and gracilis autograft. Only $1.5 \%(2 / 129)$ would attempt surgical repair. Ligament augmentation is not used by 97\% (126/129) of surgeons, and $83 \%(108 / 129)$ do not incorporate the residual ACL stump in the repair. The reasons for incorporation of the stump were given as possible help in revascularisation of the graft or prevention of synovial fluid from entering the tunnel. For a similar scenario, 60\% (86/144) would recommend ACL reconstruction for a female patient, whereas 37\% (53/144) would treat her with rehabilitation and bracing. Some $48 \%$ (68/143) would treat a recreational soccer player with ACL reconstruction or rehabilitation and bracing. For an 18 year old non-athlete, $60 \%$ (85/141) would recommend rehabilitation and bracing whereas 35\% (51/141) would perform an ACL reconstruction. For a 14 year old, 40\% (57/141) would treat with rehabilitation and bracing, 30\% (42/141) would perform an ACL reconstruction immediately, and the same percentage would perform ACL reconstruction after the physes are closed. For an 8 year old, 53\% (72/135) would recommend rehabilitation and bracing whereas $32 \%$ (44/135) would perform an ACL reconstruction after the physes close; $14 \%$ (19/135) would perform an immediate reconstruction.

In the second scenario of a 22 year old with a chronic ACL deficient knee, $83 \%$ (121/146) of surgeons would recommend ACL reconstruction; 62\% (85/137) would perform bonepatellar tendon-bone reconstruction, whereas 33\% (46/137) would prefer a semitendinosus and gracilis autograft. In the scenario of failed bracing, 98\% (145/148) recommended ACL reconstruction (fig 1 ).

Age in itself was not a factor affecting the decision to operate, or at least not for $63 \%(91 / 146)$ of surgeons. It certainly is not a factor that affects the type of surgery performed, with $82 \%(115 / 136)$ of surgeons not altering their strategy with age. Patellofemoral factors did not alter greatly the decision to operate, with $89 \%$ (129/146) of surgeons not influenced by this. The type of surgery in patients with patellofemoral arthritis was altered by $20 \%$ (27/138) of surgeons, with most opting for semitendinosus and gracilis reconstruction and avoiding bone-patellar tendon-bone reconstruction. An equivocal response was received with regard to moderate osteophyte formation with joint space narrowing, with $44 \%(63 / 144)$ taking it into account and 56\% $(81 / 144)$ not. Severe joint space narrowing leads $96 \%$ (139/ 145) of surgeons to reconsider their decision. Most surgeons would consider high tibial osteotomy or unicompartmental arthroplasty. The alignment of the knee did not appear to alter decision making in terms of proceeding to surgery: 59\% $(86 / 147)$ do not change their decision to operate on the basis of alignment, whereas 65\% (89/138) would not change the type of surgery if malalignment were present.

\section{DISCUSSION}

This survey shows the variety of approaches in the diagnosis and treatment of ACL injuries in the United Kingdom. The diagnosis of ACL injuries in the United Kingdom is still made essentially with history, clinical examination, and plain radiographs. ${ }^{11}{ }^{12}$ Arthroscopy and MRI scan is used routinely by $14 \%$ and $26 \%$ of surgeons respectively to diagnose ACL injuries. These figures may reflect the limitations of practice in the NHS set up rather than indicate the surgeons' preferences. There were comments from surgeons indicating that they would like to use MRI scans as a diagnostic tool more often if it were more easily and quickly available. It is interesting to note the use of arthroscopy as a diagnostic tool

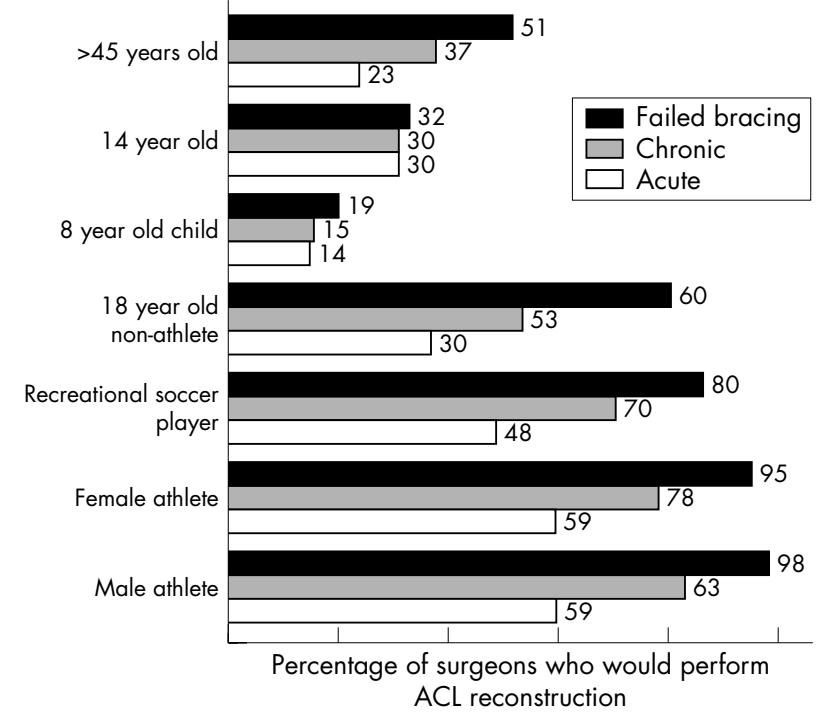

Figure 1 Effect of patient factors on the decision to perform anterior cruciate ligament $(\mathrm{ACL})$ reconstruction. 
by $14 \%$ of the surgeons, as arthroscopy is increasingly believed to have a therapeutic rather than diagnostic role in the management of ACL injuries. Over half $(55 \%)$ of the surgeons would delay surgery for a certain period of time even if the patient had a full range of movement. This would be in line with the present published evidence suggesting that patients are more likely to get arthrofibrosis if operated on in the first one to two weeks after injury. ${ }^{13}$ However, $31 \%$ of the surgeons would operate on the basis of the patient having full range of movement even if it meant operating immediately. The vast majority of surgeons do not use any ligament augmentation. Some (16\%) incorporate the remnants of the ACL stump, the most common reasons being supposed help with revascularisation and possible help with proprioception. This may represent the trend in the debate over the benefits and drawbacks of using the ACL remnants in the reconstruction. There is currently no conclusive evidence to suggest that one technique is better than the other. The sex of the patient does not alter the management, with a similar percentage of surgeons opting for ACL reconstruction for a similarly aged female patient.

There is considerable variation in the management of ACL injuries in children and adolescents. Only $14 \%$ of the surgeons who responded to the questionnaire would operate on an 8 year old with an acute ACL rupture; 32\% would wait until the physes have closed, and 53\% would treat these patients conservatively. In an adolescent patient with an acute ACL injury, 30\% would operate soon, 30\% would wait until the physes are closed, and $40 \%$ would treat with rehabilitation and/or braces. Only 15\% would operate on an 8 year old with chronic ACL rupture. This is probably because of the potential risk of injury to the growth plate resulting in growth disturbance. Some of the recently published studies, on the other hand, suggest that children with ACL deficient knees tend to do considerably worse with conservative treatment. ${ }^{14-16}$ Most surgeons would not recommend surgery in the presence of severe joint space narrowing. Anatomical malalignment is not considered by $59 \%$ to be important in decisions about surgery.

Although this was a nationwide survey of ACL injury management practice in the United Kingdom, this study has some limitations. Only the BOA members who are also members of one of the specialist societies related to knee surgery-BOSTA and BASK members-were sent this survey. We do not know whether this population provides a true representation of the current practice of ACL injury management in United Kingdom. The response rate was $60 \%$, which should be accepted as a reasonable sample of the population surveyed. The non-responders were not in any known way different from the responders.

A similar study was performed in Canada in 1996. ${ }^{9}$ The results of the two studies are comparable. There are, however, some differences. For example, we found a lower rate of ACL stump incorporation ( $16 \% \vee 40 \%)$ and a lower recommendation for surgery in children with acute ACL ruptures (14\% $v$ $30 \%$ ). The continued use of arthroscopy as a purely diagnostic tool needs to be reviewed. This survey provides an interesting insight into the management of ACL injuries in the United Kingdom and in some cases reasons for certain practices. These data should not be misconstrued as recommended practice. In particular, we stress that current or most up to date practice is not necessarily good practice. For example, the indications for reconstruction of the ACL are still not well codified, and there is a considerable difference between North America, Australia, Continental Europe, and the United Kingdom. In North America, Australia, and Continental Europe, the indication for operative reconstruction seems to be more and more the tear of the ACL-that is, just the anatomical lesion-whereas functional instabilitythat is, the symptom produced by a torn ACL and present in only a proportion of patients with a torn ACL-is probably a more appropriate indication for surgery. ${ }^{1}{ }^{12}$ This study may be extended to other English speaking nations to collate data on management of these injuries and it may be repeated to assess the effects of new evidence.

\section{ACKNOWLEDGEMENTS}

Many thanks are given to Ms Lisa Horne, Department of Clinical Audit, North Staffordshire Hospital, for her help in drafting and analysing the questionnaires.

\section{Authors' affiliations}

B Kapoor, D J Clement, N Maffulli, Keele University School of Medicine, Hartshill, UK

A Kirkley, University of Western Ontario, Canada

\section{REFERENCES}

1 Bollen SR, Scott BW. Rupture of the anterior cruciate ligament: a quiet epidemic? Injury 1996;27:407-9.

2 Caborn DNM, Johnson B. The natural history of the anterior cruciate ligament deficient knee. Clin Sports Med 1993;12:625-36.

3 Learmonth D. Incidence and diagnosis of anterior cruciate ligament injuries in the accidents and emergency department. Injury 1991;22:287-90.

4 Aglietti P, Buzzi R, D’Andria S, et al. Patellofemoral problems after intraarticular anterior cruciate ligament reconstruction. Clin Orthop 1993;288: 195-204.

5 Buss D, Min R, Skyhar MJ, et al. Nonoperative treatment of acute anterior cruciate ligament injuries in a selected group of patients. Am J Sports Med 1995;23: 160-5.

6 Fernando B, Bastos MN, Sacristan CG. Semitendinosus Kennedy ligament augmentation device anterior cruciate ligament reconstruction. Clin Orthop 1992;283:21-33.

7 Kumar K, Maffulli N. The ligament augmentation device: an historical perspective. Arthroscopy 1999; 15:422-32.

8 Mirza F, Mai DD, Kirkley A, et al. Management of injuries to the anterior cruciate ligament: results of a survey of orthopaedic surgeons in Canada. Clin J Sport Med 2000;10:85-8.

9 Dillman DA. Mail and telephone surveys: the total design method. New York: John Wiley and Sons, 1978.

10 Clark CR, Heckman JD. Volume versus outcomes in orthopaedic surgery: a proper perspective is paramount. J Bone Joint Surg [Am] 2001;83:1619-21.

11 Johnson D, Warner J. Diagnosis for anterior cruciate ligament surgery. Clin Sports Med 1993;12:671-84.

12 Maffulli N, Binfield PM, King JB, et al. Acute haemarthrosis of the knee in athletes. A prospective study of 106 cases. J Bone Joint Surg $[\mathrm{Br}]$ 1993;75:945-9.

13 Shelbourne KD, Wilcknes J, Mollabashy A, et al. Arthrofibrosis in acute anterior cruciate ligament reconstruction: the effect of timing of reconstruction and rehabilitation. Am J Sports Med 1991;19:332-6.

14 Ian KY, Kirkley A, Fowler PJ, et al. The outcome of operatively treated anterior cruciate ligament disruptions in the skeletally immature child. Arthroscopy 1997; 13:627-34.

15 Aichroth PM, Patel DV, Zorilla P. The natural history and treatment of rupture of the anterior cruciate ligament in children and adolescents. J Bone Joint Surg [Br] 2002;84:38-41.

16 Graf BK, Lange RH, et al. Anterior cruciate ligament tears in skeletally immature patients: meniscal pathology at presentation and after attempted conservative treatment. Arthroscopy 1992;8:229-33. 